\title{
A Description of Iterative Reflections of Monads (Extended Abstract)
}

\author{
Jiří Adámek ${ }^{1}$, Stefan Milius ${ }^{1}$, and Jiří Velebil ${ }^{2, \star}$ \\ 1 Institute of Theoretical Computer Science, Technical University, \\ Braunschweig, Germany \\ adamek@iti.cs.tu-bs.de, mail@stefan-milius.eu \\ 2 Faculty of Electrical Engineering, Czech Technical University of Prague, \\ Czech Republic \\ velebil@math.feld.cvut.cz
}

\begin{abstract}
For ideal monads in Set (e.g. the finite list monad, the finite bag monad etc.) we have recently proved that every set generates a free iterative algebra. This gives rise to a new monad. We prove now that this monad is iterative in the sense of Calvin Elgot, in fact, this is the iterative reflection of the given ideal monad. This shows how to freely add unique solutions of recursive equations to a given algebraic theory. Examples: the monad of free commutative binary algebras has the monad of binary rational unordered trees as iterative reflection, and the finite list monad has the iterative reflection given by adding an absorbing element.
\end{abstract}

\section{Introduction}

The semantics of recursive definitions is a topic at the heart of theoretical computer science. Iterative theories of Calvin Elgot are a well-established formalism in which recursive equation systems can be solved. So far, iterative theories were considered over arbitrary signatures [910] or arbitrary endofunctors 3] but without studying the effect of equational laws on given operations. For example, Elgot et al. described in [10] the free iterative theory on a signature $\Sigma$ as the theory $\mathbb{R}_{\Sigma}$ of all rational $\Sigma$-trees (that is, $\Sigma$-trees with only finitely many subtrees up to isomorphism). The free iterative theory can be thought of as the closure of the theory formed by all $\Sigma$-terms under unique solutions of recursive equations. In our present paper we attend to the influence that equations have on iteration. This topic is relevant e.g. for process algebra where processes are defined recursively and operations on processes typically satisfy equational laws such as associativity, commutativity or idempotency. Let us consider the simple case of one binary operation: by the above result the free iterative theory is the theory of rational binary trees. What happens if the operation is required to be commutative? The answer is simple: the free iterative theory consists of all rational non-ordered binary trees. This has been known before since commutativity can be expressed by working with algebras for an endofunctor $H$, and in

\footnotetext{
* The third author acknowledges the support of the Grant Agency of the Czech Re-
} public under the Grant No. 201/06/664. 
that case the free iterative theory was described in [2] as follows: one applies the given equations to rational $\Sigma$-trees possibly infinitely often. Next question: what happens if the given binary operation is required to be associative? That is, the theory we start with is the theory of finite lists. It follows from our results in the present paper that the free iterative theory is just the extension of the finite-list theory by a single absorbing element. (Informally, for every infinite binary tree one gets, by applying the associative law infinitely often, the complete binary tree viz. the unique solution of $x \approx x \cdot x$.) The same answer is true for a commutative and associative binary operation, in other words, for the finite-bag theory. Last question: what about an idempotent binary operation? We cannot provide an answer to this question because the corresponding theory is not ideal, and we have only iterative reflections for ideal theories - in fact, the question makes no sense for general theories.

We are going to work with finitary monads $\mathbb{M}$ rather than equational theories - recall that the underlying functor $M$ of the monad assigns to every set $X$ the free algebra $M X$ on $X$ for the given equational theory, and that the inclusion of generators forms a natural transformation $\eta:$ Id $\rightarrow M$. Elgot 9 ] called $\mathbb{M}$ ideal if $M$ is a coproduct of Id and a subfunctor $M^{\prime}$ such that $\eta$ is one of the coproduct injections, and the monad multiplication $\mu: M M \rightarrow M$ has a restriction to $\mu^{\prime}: M^{\prime} M \rightarrow M^{\prime}$; in the language of theories that means that the presentation of $M$ by operations modulo equations is such that the property of a term not being equivalent to a variable is preserved by substitution. Commutativity and associativity of a binary operation are examples of equational specifications yielding ideal monads, idempotency is not.

We already know that every ideal monad has an iterative reflection; this states in category-theoretic terms that unique solutions of guarded recursive equations can be added freely to the given monad. This was proved in [5] under much less restrictive side conditions than those required below. However, a concrete description of the iterative reflection was missing: we proved that for a given ideal monad $\mathbb{M}$ every object $X$ generates a free iterative algebra $\widehat{M} X$, and thus, we obtain a new monad $\widehat{\mathbb{M}}$. Here we prove that $\widehat{\mathbb{M}}$ is iterative and that it is the desired iterative reflection of $\mathbb{M}$.

Although the statement "the iterative reflection is the monad of free iterative algebras" may sound almost tautological, we have not found an easy proof. In fact, the proof presented in our paper is not only technically involved, it also requires at one point that every strong epimorphism be split- this forces us to restrict our attention to monads in categories such as sets or vector spaces. In contrast, the existence of iterative reflections was proved for ideal monads in all extensive locally finitely presentable categories, see [5].

In this extended abstract we omit most of the technical details and provide only sketches of proofs. The full details can be found in [6].

\section{$2 \quad$ Ideal and Iterative Monads}

In this section we recall the concepts introduced by Calvin Elgot 9 in the language of monads in lieu of algebraic theories used originally. Recall first that 
a functor is called finitary if it preserves filtered colimits. Throughout this section $\mathbb{M}=(M, \eta, \mu)$ denotes a finitary monad on a category $\mathcal{A}$ (that is, the underlying endofunctor $M$ is finitary). Recall further that given another monad $\overline{\mathrm{M}}=(\bar{M}, \bar{\eta}, \bar{\mu})$, a monad morphism is a natural transformation $h: M \rightarrow \bar{M}$ such that $h \cdot \eta=\bar{\eta}$ and $h \cdot \mu=\bar{\mu} \cdot(h * h)$.

Assumption 2.1. Throughout the paper we assume that the base category $\mathcal{A}$ is locally finitely presentable and has split strong epimorphisms. More detailed, we assume that (1) $\mathcal{A}$ has colimits, (2) for every strong epimorphism $e: X \rightarrow Y$ there exists $m: Y \rightarrow X$ with $e \cdot m=$ id (where "strong" means the diagonal fill-in property w.r.t. all monomorphisms), and (3) $\mathcal{A}$ has a set $\mathcal{A}_{\mathrm{fp}}$ of finitely presentable objects (i.e., their hom-functors are finitary) whose closure under filtered colimits is all of $\mathcal{A}$.

For example, the categories of sets and many-sorted sets satisfy the above assumptions, with $\mathcal{A}_{\mathrm{fp}}$ formed by all finite sets. For every field $K$ the category $\operatorname{Vec}_{K}$ of vector spaces on $K$ also satisfies (1)-(3) and $\mathcal{A}_{\mathrm{fp}}$ is formed by the finitedimensional spaces. Finally, every algebraic lattice $\mathcal{A}$ considered as a category fulfills $(1)-(3)$; the finitely presentable objects are the compact elements.

Notation 2.2. The category of (Eilenberg-Moore) algebras for the monad $\mathbb{M}$ is denoted by $\mathcal{A}^{\mathbb{M}}$. Recall that its objects are algebras $a: M A \rightarrow A$ for the functor $M$ such that $a \cdot \eta_{A}=$ id and $a \cdot M a=a \cdot \mu_{A}$. And the morphisms of $\mathcal{A}^{\mathbb{M}}$ are the usual $M$-algebra homomorphisms, i. e. $h$ is a homomorphism from an algebra $(A, a)$ to $(B, b)$ if $h \cdot a=b \cdot M h$.

Definition 2.3. (C. Elgot [9]) An ideal monad consists of a finitary monad $\mathbb{M}=(M, \eta, \mu)$, a finitary subfunctor $m: M^{\prime} \hookrightarrow M$ such that $M=M^{\prime}+$ Id with injections $m$ and $\eta$, and a natural transformation $\mu^{\prime}: M^{\prime} M \rightarrow M^{\prime}$ such that the square below commutes:

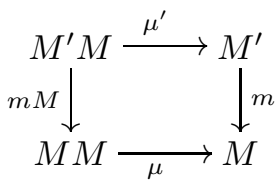

Definition 2.4. Let $\mathbb{M}$ be an ideal monad.

(1) By a (finitary) equation morphism we mean a morphism $e: X \rightarrow M(X+$ $Y$ ), where $X$ is a finitely presentable object (of variables) and $Y$ is an arbitrary object (of parameters).

(2) We call e guarded provided that it factorizes through the summand $M^{\prime}(X+$ $Y)+Y$ of $M(X+Y)=M^{\prime}(X+Y)+X+Y$ :

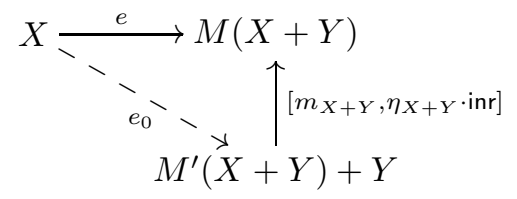


Remark 2.5. Recall that if $\mathcal{A}=$ Set, then for every finitary monad $\mathbb{M}$ there exists an equational presentation such that $\mathbb{M}$ is the associated free-algebra monad. That is, for every set $Z$ we can consider $M Z$ as the set of all terms with the free variables in $Z$ modulo the given equations.

(1) If $X=\left\{x_{1}, \ldots, x_{n}\right\}$ then the equation morphism $e$ can be regarded as the system of recursive equations

$$
\begin{aligned}
x_{1} & \approx t_{1}\left(x_{1}, \ldots, x_{n}, p_{1}, \ldots, p_{k}\right) \\
& \vdots \\
x_{n} & \approx t_{n}\left(x_{1}, \ldots, x_{n}, p_{1}, \ldots, p_{k}\right)
\end{aligned}
$$

whose right-hand sides $t_{i}=e\left(x_{i}\right)$ are $\mathbb{M}$-terms in the variables from $X$ and parameters $p_{1}, \ldots, p_{k} \in Y$.

(2) The concept of a guarded equation morphism forbids equations such as $x_{1} \approx x_{1}$. Evelyn Nelson [14] introduced iterative algebras for a signature in which guarded systems of equations have unique solutions - see also the related concept by Jerzy Tiuryn [15]:

Definition 2.6. We say that the algebra $(A, a)$ for the ideal monad $\mathbb{M}$ is iterative provided that every guarded equation morphism $e: X \rightarrow M(X+A)$ with parameter object $Y=A$ has a unique solution, i. e., a unique morphism $e^{\dagger}: X \rightarrow A$ for which

$$
e^{\dagger}=a \cdot M\left[e^{\dagger}, \operatorname{id}_{A}\right] \cdot e .
$$

Remark 2.7. For every ideal monad $\mathbb{M}$ the following was proved in $[5]$ :

(1) Iterative algebras form a full subcategory of $\mathcal{A}^{\mathbb{M}}$; the reason why we consider the usual homomorphisms as the "right" morphisms of iterative algebras is that homomorphisms automatically preserve solutions.

(2) Every object $X$ generates a free iterative $\mathbb{M}$-algebra which we denote by $\widehat{M} X$ with the structure and the universal arrow given by $\rho_{X}: M \widehat{M} X \rightarrow \widehat{M} X$ and $\widehat{\eta}_{X}: X \rightarrow \widehat{M} X$. In other words, the forgetful functor of the category of iterative $\mathbb{M}$-algebras has a left adjoint $X \mapsto\left(\widehat{M} X, \rho_{X}\right)$.

(3) We obtain a new monad $\widehat{\mathbb{M}}=(\widehat{M}, \widehat{\eta}, \widehat{\mu})$ and a monad morphism $\kappa: \mathbb{M} \rightarrow \widehat{\mathbb{M}}$ with the components $\kappa_{X}=\rho_{X} \cdot M \widehat{\eta}_{X}$. We also proved that the ideal monad $\mathbb{M}$ has an iterative reflection-and in the present paper we prove that this is $\kappa: \mathbb{M} \rightarrow \widehat{\mathbb{M}}$.

In [5] we assumed that the base category is extensive, and therefore we worked with ideal (rather than guarded) equation morphisms. However, all the results remain valid under our present assumption. In particular, our proof of the existence of an iterative reflection (Theorem 2.13) contained in Remark 2.14 uses split epimorphisms and does not use extensivity. The proof is based on the fact (proved in [5) that iterative algebras are closed under limits - by inspecting the proof one sees immediately that this is true for iterativity based, as in Definition 2.6 above, on guarded equation morphisms. Also extensivity plays no rôle here.

Examples 2.8. We mention some well-known ideal monads $\mathbb{M}$ in Set for which a description of the free iterative $\mathbb{M}$-algebra $\widehat{M} X$ on $X$ is known, see [5]. 
(1) The monad $M X=X^{*}$ of finite lists in $X$ whose $\mathbb{M}$-algebras are monoids. A free iterative algebra is described in [5]: one adds to $X^{*}$, the monoid of finite lists with concatenation, an absorbing element $\perp$; this means that the binary operation is extended by $w \perp=\perp=\perp w$ for all lists $w$. Shortly: $\widehat{M} X=X^{*}+\{\perp\}$.

(2) The monad $\mathbb{M}$ assigning to a set $X$ the set of finite trees with leaves labelled in $X$. The $\mathbb{M}$-algebras are precisely the $\Sigma$-algebras for the signature $\Sigma$ of one $n$-ary operation symbol for every natural number $n=1,2,3, \ldots$. The free iterative algebra was described by Evelyn Nelson [14] using the concept of rational tree of Susanna Ginali [12, meaning a tree with finitely many subtrees up to isomorphism: $\widehat{M} X$ consists of all finitely branching rational trees with leaves labelled in $X$.

(3) Analogously, let $M X$ consist of all finite non-ordered binary trees on $X$, so $\mathbb{M}$ is the monad of commutative binary algebras. In this case $\widehat{M} X$ consists of all rational non-ordered binary trees on $X$, see [2].

(4) The monad $\mathbb{M}$ assigning to a set $X$ the set of finite bags (= multisets) in $X$ has as algebras the commutative monoids. A free iterative algebra, as proved in [14], is $\widehat{M} X=M X+\{\perp\}$ where $\perp$ is an absorbing element.

(5) The monad $M X=X \times \Sigma^{*}$ of free unary algebras with operations from the set $\Sigma$ has the free iterative algebras $\widehat{M} X=\left(X \times \Sigma^{*}\right)+\Sigma^{p}$ where $\Sigma^{p}=$ $\Sigma^{*}\left(\Sigma^{*}\right)^{\omega}$ are the words in $\Sigma$ which are eventually periodic, see 14. For example, $\widehat{M} X=X \times \mathbb{N}+1$ in case $\Sigma=\{0\}$.

(6) The monad $M X=X \times \mathbb{N}^{\Sigma}$ of free unary algebras with commuting operations from the set $\Sigma$ (i.e., $a(b(x))=b(a(x))$ for all $a, b \in \Sigma)$ has the free iterative algebras $\widehat{M} X=\left(X \times \mathbb{N}^{\Sigma}\right)+\{\perp\}$ where $\perp$ is a fixed point of all operations. In case of two operations this was pointed out to us by Bruno Courcelle, and a detailed proof is presented in 5. The general case is completely analogous.

(7) For every finitary endofunctor $H$ we have described in 3 a free iterative monad on $H$ as the monad of free iterative algebras for the functor $H$. Moreover, a free iterative $H$-algebra on the object $Y$ is given as the colimit of all finite coalgebras for $H(-)+Y$. We know from Michael Barr 8 that $H$-algebras are precisely the monadic algebras for the free monad $\mathbb{M}$ on $H$, and it is not difficult to prove that iterative algebras for the functor $H$ in the sense of [3] (see Definition 2.5 and Theorem 4.4) are precisely the iterative algebras for $\mathbb{M}$ as defined in 2.6. Thus, the free iterative monad on $H$ is the monad $\widehat{\mathbb{M}}$. Special cases include (2) above for $H X=\coprod_{n \in \mathbb{N}} X^{n}$, (3) for $H X=\{\{x, y\} \mid x, y \in X\}$ and (5) above for $H X=X \times \Sigma$. In [3] we called $\widehat{\mathbb{M}}$ the rational monad of $H$.

Remark 2.9. In contrast to Example 2.8(4), the monad $\mathcal{P}_{\text {fin }}$ of finite subsets is not ideal: consider $x \neq y$ in $X$ and a function $f: X \rightarrow X^{\prime}$ with $f(x)=f(y)$. Then $\{x, y\} \in \mathcal{P}_{\text {fin }} X \backslash \eta_{X}[X]$ but $\mathcal{P}_{\text {fin }} f\{x, y\} \in \eta_{X^{\prime}}\left[X^{\prime}\right]$.

Definition 2.10. [9] An ideal monad $\mathbb{M}$ is called iterative if every guarded equation morphism $e: X \rightarrow M(X+Y)$ has a unique solution w.r.t. $\mathbb{M}$, which means a morphism $e^{\dagger}: X \rightarrow M Y$ such that the equation $e^{\dagger}=\mu_{Y} \cdot M\left[e^{\dagger}, \eta_{Y}\right] \cdot$ e holds. 
Proposition 2.11. [5] An ideal monad $\mathbb{M}$ is iterative if and only if all of its free algebras $\left(M X, \mu_{X}\right)$ are iterative algebras.

Example 2.12. The monad $\widehat{M} X=X \times \mathbb{N}+1$ of $2.8(5)$ is iterative: its free algebras are the free algebras on one unary operation $\sigma$ extended by a unique fixed point of $\sigma$. In general, an algebra for $\widehat{\mathbb{M}}$ is an algebra with a unary operation with a chosen fixed point of that operation, see [4. So there exist many algebras for $\widehat{\mathbb{M}}$ that are not iterative, e.g., all those with more than one fixed point for $\sigma$. In fact, in an iterative algebra the equation $x \approx \sigma(x)$ must have a unique solution.

Definition 2.13. Suppose we have two ideal monads $\mathbb{M}=\left(M, \eta, \mu, M^{\prime}, m, \mu^{\prime}\right)$ and $\overline{\mathrm{M}}=\left(\bar{M}, \bar{\eta}, \bar{\mu}, \bar{M}^{\prime}, \bar{m}, \bar{\mu}^{\prime}\right)$. By an ideal monad morphism we understand $a$ monad morphism $h:(M, \eta, \mu) \rightarrow(\bar{M}, \bar{\eta}, \bar{\mu})$ such that there exists a domaincodomain restriction $h^{\prime}: M^{\prime} \rightarrow \bar{M}^{\prime}$ of $h$ with $\bar{m} \cdot h^{\prime}=h \cdot m$ (which is necessarily unique, recall that $\bar{m}$ is a monomorphism).

Notation 2.14. In the category of all finitary monads on $\mathcal{A}$ we now consider (a) the non-full subcategory of all ideal monads and ideal monad morphisms, denoted by $\mathrm{FM}_{\mathrm{id}}(\mathcal{A})$ and (b) its full subcategory of all iterative monads $\operatorname{IFM}(\mathcal{A})$.

\section{A Characterization of Free Iterative Algebras}

Assumption 3.1. Throughout the rest of the paper $\mathbb{M}$ denotes an ideal monad on $\mathcal{A}$.

Remark 3.2. In 3] we described for every endofunctor $H$ of a locally finitely presentable category, the free iterative $H$-algebra on an object $Y$ as a colimit of the diagram $\mathrm{Eq}_{Y}$ of all flat equation morphisms, i. e., morphisms $e: X \rightarrow$ $H X+Y$ with $X$ finitely presentable. The connecting morphisms of that diagram $\mathrm{Eq}_{Y}$ are simply the coalgebra homomorphisms for the endofunctor $H(-)+Y$. The fact that $\mathrm{Eq}_{Y}$ is a filtered diagram whose colimit is the free iterative $H$ algebra on $Y$ turned out to be the basic step for describing the rational monad of $H$, see Example 2.8 (7). The proof of this fact was technically rather involved.

In the present section we prove the analogous result for algebras for an ideal monad $\mathbb{M}$ : we form the diagram of all guarded equation morphisms $e: X \rightarrow$ $M(X+Y)$ with $X$ finitely presentable, but unfortunately, in lieu of coalgebra homomorphisms for $M((-)+Y)$ we need more general "solution homomorphisms" here. To make sure that $\mathrm{Eq}_{Y}$ is a filtered diagram we need the restrictive side condition that strong epimorphisms split.

Notation 3.3. Given an equation morphism $e: X \rightarrow M(X+Y)$, every morphism $h: Y \rightarrow Y^{\prime}$ yields a new equation morphism (by changing parameters)

$$
h \bullet e \equiv X \stackrel{e}{\longrightarrow} M(X+Y) \stackrel{M(X+h)}{\longrightarrow} M\left(X+Y^{\prime}\right) \text {. }
$$

In particular, use the universal arrow $\widehat{\eta}_{Y}: Y \rightarrow \widehat{M} Y$ of Remark 2.7 to turn every "abstract" equation morphism $e: X \rightarrow M(X+Y)$ into a "concrete" equation morphism $\widehat{\eta}_{Y} \bullet e: X \rightarrow M(X+\widehat{M} Y)$ in the free iterative algebra $\left(\widehat{M} Y, \rho_{Y}\right)$. 
The latter has, whenever $e$ is guarded, a unique solution in $\widehat{M} Y$ which, by abuse of notation, we denote by $e^{\dagger}: X \rightarrow \widehat{M} Y$. Thus, for every guarded equation morphism $e: X \rightarrow M(X+Y)$ we define $e^{\dagger}$ by

$$
e^{\dagger}=\rho_{Y} \cdot M\left[e^{\dagger}, \widehat{\eta}_{Y}\right] \cdot e .
$$

Definition 3.4. Let $e: X \rightarrow M(X+Y)$ and $f: X^{\prime} \rightarrow M\left(X^{\prime}+Y\right)$ be guarded equation morphisms. By a solution homomorphism is meant a morphism $h$ : $X \rightarrow X^{\prime}$ in $\mathcal{A}$ for which the equation $f^{\dagger} \cdot h=e^{\dagger}: X \rightarrow \widehat{M Y}$ holds.

Notation 3.5. For every object $Y$ we denote by $\mathrm{EQ}_{Y}$ the category of all guarded equation morphisms with parameter object $Y$ and all solution homomorphisms.

We also denote by $\mathrm{Eq}_{Y}: \mathrm{EQ}_{Y} \rightarrow \mathcal{A}$ the forgetful functor assigning to $e: X \rightarrow$ $M(X+Y)$ the object $X$.

Example 3.6. Whenever $h: X \rightarrow X^{\prime}$ is a coalgebra homomorphism, i. e., whenever we have $f \cdot h=M(h+Y) \cdot e$, then $h$ is a solution homomorphism. Indeed, $f^{\dagger} \cdot h=e^{\dagger}$ follows from the uniqueness of solutions since $f^{\dagger} \cdot h$ solves $e$. To see this consider the diagram below:

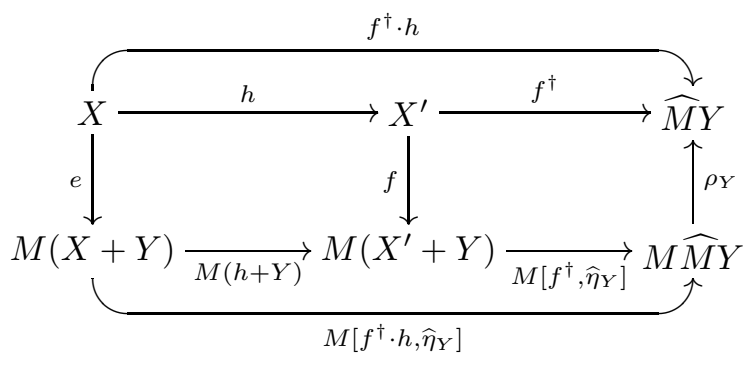

The right-hand square commutes by (3.1), the left-hand one by assumption and the upper and lower parts obviously do. So the outside of the diagram commutes, showing that $f^{\dagger} \cdot h$ is a solution of $e$ as desired.

Remark 3.7. In the coalgebraic construction of the free iterative monad on an endofunctor $H$ in [3] we used the category $\mathrm{EQ}_{Y}$ of all flat equation morphisms. This category is trivially filtered because it is closed under finite colimits in the category of all coalgebras, and so the corresponding forgetful functor $\mathrm{Eq}_{Y}$ is a filtered diagram whose colimit yields the object assignment of the desired free iterative monad on $H$.

Our present setting is more subtle: here we cannot work with coalgebra homomorphism (for $M((-)+Y)$ ) because they are insufficient to relate all equations with the same solution in the corresponding diagram. To see this we consider the example of a signature with one binary operation $*$. The associated free monad on that signature is the finite binary tree monad $\mathbb{M}$. Now let, just in this example, $\mathrm{EQ}_{Y}^{\prime}$ denote the category of guarded equation morphisms and coalgebra homomorphisms. Consider the two recursive equations (trees are written as terms here)

$$
x \approx x * y \quad \text { and } \quad x \approx(x * y) * y
$$


which give rise to two different equation morphism $e, f:\{x\} \rightarrow M(\{x\}+\{y\})$. These two equations specify the same rational binary tree on $\{y\}$ :

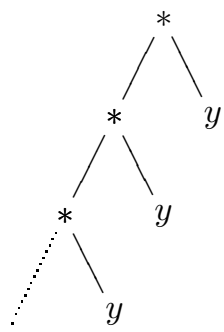

However, the above two equations will lead to two distinct elements in the colimit of the diagram given by the forgetful functor on $\mathrm{EQ}_{Y}^{\prime}$ - this is due to the fact that any morphism in $\mathrm{EQ}_{Y}^{\prime}$ preserves the height of the binary trees on the right-hand side of recursive equations.

Lemma 3.8. The category $\mathrm{EQ}_{Y}$ is filtered.

Theorem 3.9. The free iterative algebra $\widehat{M} Y$ is a filtered colimit of the diagram $\mathrm{Eq}_{Y}: \mathrm{EQ}_{Y} \rightarrow \mathcal{A}$ of all guarded equation morphisms in $Y$, shortly, $\widehat{M} Y=$ colim $\mathrm{Eq}_{Y}$.

The overall structure of the proof is somewhat similar to the structure of the proof of Theorem 3.3 in [3]. However, the technical details are different and more involved.

Sketch of Proof. We denote by $\widetilde{M} Y$ a colimit of the filtered diagram $\mathrm{Eq}_{Y}$ in $\mathcal{A}$ with colimit morphisms $e^{\sharp}: X \rightarrow \widetilde{M} Y$ for all $e: X \rightarrow M(X+Y)$ in $\mathrm{EQ}_{Y}$. We first turn $\widetilde{M} Y$ into an $\mathbb{M}$-algebra. Recall that since $\mathcal{A}$ is locally finitely presentable, the object $M \widetilde{M} Y$ is a colimit of the canonical diagram of all morphisms $p$ : $P \rightarrow M \widetilde{M} Y$ with $P$ finitely presentable. Thus, we can define a morphism $\widetilde{\rho}_{Y}$ : $M \widetilde{M} Y \rightarrow \widetilde{M} Y$ by specifying its composites $\widetilde{\rho}_{Y} \cdot p$ with any $p: P \rightarrow M \widetilde{M} Y$ with $P$ finitely presentable. Since $M$ is finitary and $X$ is finitely presentable, for every $p$ there exists $e: X \rightarrow M(X+Y)$ in $\mathrm{EQ}_{Y}$ and a morphism $p_{0}: P \rightarrow M X$ such that $p=M e^{\sharp} \cdot p_{0}$. Now denote by $\llbracket p, e \rrbracket$ the guarded equation morphism

$$
P+X \stackrel{\left[p_{0}, \eta\right]}{\longrightarrow} M X \stackrel{M e}{\longrightarrow} M M(X+Y) \stackrel{\mu}{\longrightarrow} M(X+Y) \stackrel{M \mathrm{inr}}{\longrightarrow} M(P+X+Y) .
$$

One then proves that the morphisms $\llbracket p, e \rrbracket^{\sharp} \cdot$ inl $: P \rightarrow \widetilde{M} Y$ are independent of the choice of $p_{0}$, and that they form a cocone of the canonical diagram. Thus, there exists a unique morphism $\widetilde{\rho}_{Y}: M \widetilde{M} Y \rightarrow \widetilde{M} Y$ such that $\widetilde{\rho}_{Y} \cdot p=\llbracket p_{0}, e \rrbracket^{\sharp} \cdot$ inl holds for all $p$ as above. One can now prove that $\widetilde{\rho}_{Y}$ is the structure morphism of an M-algebra.

Next consider for a finitely presentable object $Y$ the trivial guarded equation morphism $\eta_{Y+Y} \cdot \operatorname{inr}: Y \rightarrow M(Y+Y)$ and let $\widetilde{\eta}_{Y}=\left(\eta_{Y+Y} \cdot \text { inr }\right)^{\sharp}: Y \rightarrow \widetilde{M} Y$. 
Observe that the morphisms $e^{\dagger}: X \rightarrow \widehat{M} Y\left(e\right.$ in $\left.\mathrm{EQ}_{Y}\right)$ form a cocone of the diagram $\mathrm{Eq}_{Y}$, thus, there exists a unique morphism $i: \widetilde{M} Y \rightarrow \widehat{M} Y$ such that $i \cdot e^{\sharp}=e^{\dagger}$ holds for all $e$ in $\mathrm{EQ}_{Y}$. One now proves that $i:\left(\widetilde{M} Y, \widetilde{\rho}_{Y}\right) \rightarrow\left(\widehat{M} Y, \rho_{Y}\right)$ is a homomorphism of $\mathbb{M}$-algebras such that $i \cdot \widetilde{\eta}_{Y}=\widehat{\eta}_{Y}$.

Using this property of $i$ one then verifies that for each finitely presentable object $Y$ and for each $e: X \rightarrow M(X+Y)$ in $\mathrm{EQ}_{Y}$ the colimit injection $e^{\sharp}: X \rightarrow$ $\widetilde{M} Y$ is the unique morphism such that $e^{\sharp}=\widetilde{\rho}_{Y} \cdot M\left[e^{\sharp}, \widetilde{\eta}_{Y}\right] \cdot e$. And this fact is the crucial ingredient for the verification that the $\mathbb{M}$-algebra $\left(\widetilde{M} Y, \widetilde{\rho}_{Y}\right)$ is iterative.

For this verification suppose that $e: X \rightarrow M(X+\widetilde{M} Y)$ is a guarded equation morphism with a factor $e_{0}: X \rightarrow M^{\prime}(X+\widetilde{M} Y)+\widetilde{M} Y$. Since $M^{\prime}$ is finitary, the object $M^{\prime}(X+\widetilde{M} Y)+\widetilde{M} Y$ is a colimit of $M^{\prime}\left(X+\mathrm{Eq}_{Y}\right)+\mathrm{Eq}_{Y}$. Since $X$ is finitely presentable, we can choose some equation morphism $f: V \rightarrow M(V+Y)$ in $\mathrm{EQ}_{Y}$ and a factorization $w^{\prime}: X \rightarrow M^{\prime}(X+V)+V$ such that $e_{0}=\left(M^{\prime}\left(X+f^{\sharp}\right)+f^{\sharp}\right) \cdot w^{\prime}$. For $w=\left[m_{X+V}, \eta_{X+V} \cdot \mathrm{inr}\right] \cdot w^{\prime}$ define the equation morphism $\bar{e}$ by

$$
X+V \stackrel{[w, \eta \cdot \mathrm{inr}]}{\longrightarrow} M(X+V) \stackrel{M(\eta+f)}{\longrightarrow} M(M X+M(V+Y)) \stackrel{\mu \cdot M \text { can }}{\longrightarrow} M(X+V+Y) .
$$

It is not difficult to prove that $\bar{e}$ is guarded, whence an object of $\mathrm{EQ}_{Y}$. Now let $e^{\dagger}=\bar{e}^{\sharp} \cdot$ inl $: X \rightarrow \widetilde{M} Y$. One then verifies that $e^{\dagger}$ is a unique solution of $e$ in the algebra $\widetilde{M} Y$.

Since $\widehat{M} Y$ is a free iterative algebra on $Y$ we obtain a unique $\mathbb{M}$-algebra homomorphism $j: \widehat{M} Y \rightarrow \widetilde{M} Y$ such that $j \cdot \widehat{\eta}_{Y}=\widetilde{\eta}_{Y}$. Then we immediately have $i \cdot j=\mathrm{id}$, and to see that $j \cdot i=\mathrm{id}$ it is sufficient to establish $j \cdot i \cdot e^{\sharp}=e^{\sharp}$ for all $e$ in $\mathrm{EQ}_{Y}$. This completes the proof for finitely presentable objects $Y$, and the result also readily extends to arbitrary objects $Y$ by using the canonical diagram with colimit $Y$.

\section{Rational Equation Morphisms}

In this section we prove that iterative algebras have a stronger property of solving equations than stated in their definition. More precisely, it is our goal to show that every iterative algebra for $\mathbb{M}$ is also an iterative algebra for $\widehat{\mathbb{M}}$. As an example consider the monad $\mathbb{M}$ of finite binary trees, for which an algebra is a set $A$ with a binary operation. The algebra $A$ is iterative iff every guarded system of equations

$$
x_{i} \approx t_{i}\left(x_{1}, \ldots, x_{n}, a_{1}, \ldots, a_{k}\right) \quad i=1, \ldots, n
$$

where each $t_{i}$ is a finite binary tree on $\left\{x_{i} \mid i=1, \ldots n\right\}+\left\{a_{j} \mid j=1, \ldots, k\right\}$ has a unique solution. However, in lieu of finite trees we can as well take rational infinite trees on the right-hand sides. That is, in lieu of equation morphisms of the form $e: X \rightarrow M(X+A)$ we are allowed to consider all $e: X \rightarrow \widehat{M}(X+A)$, where $\widehat{\mathbb{M}}$ is the monad of free iterative $\mathbb{M}$-algebras (as constructed in Section 3 ).

Definition 4.1. By a rational equation morphism is meant a morphism $: X \rightarrow$ $\widehat{M}(X+Y)$ with $X$ finitely presentable. 
The concept of a solution in an iterative $\mathbb{M}$-algebra is based on the following

Notation 4.2. For an iterative $\mathbb{M}$-algebra $(A, a)$ we denote by $\widehat{a}: \widehat{M} A \rightarrow A$ the unique homomorphism extending the identity:

$$
\widehat{a} \cdot \rho_{A}=a \cdot M \widehat{a} \quad \text { and } \quad \widehat{a} \cdot \widehat{\eta}_{A}=\mathrm{id} .
$$

This is just the counit of the adjunction between the category of iterative algebras for $\mathbb{M}$ and the category $\mathcal{A}$. In other words, $(A, \widehat{a})$ is the corresponding algebra for the monad $\widehat{\mathbb{M}}$. In particular, for the free iterative algebras $\left(\widehat{M} Y, \rho_{Y}\right)$ of Theorem 3.9 the corresponding homomorphism $\widehat{\rho_{Y}}$ is the monad multiplication $\widehat{\mu}_{Y}: \widehat{M} \widehat{M} Y \rightarrow \widehat{M} Y$.

Definition 4.3. By a solution of a rational equation morphism $e: X \rightarrow \widehat{M}(X+$ $A)$ in an iterative $\mathbb{M}$-algebra $(A, a)$ is meant a morphism $e^{\ddagger}$ such that $e^{\ddagger}=$ $\widehat{a} \cdot \widehat{M}\left[e^{\ddagger}, A\right] \cdot e$ holds.

Remark 4.4. In order to state the theorem about unique solutions of rational equation morphisms $e$, we need to introduce the concept of $e$ being guarded. This would be easy if we knew that the monad $\widehat{\mathbb{M}}$ is ideal. Although this is actually true, and we prove this below (see Theorem [5.5), we are in no position for proving this now. In lieu of the desired equality $\widehat{M}=\widehat{M}^{\prime}+\mathrm{Id}$, we will now simply introduce a (seemingly arbitrary) subfunctor $\widehat{m}: \widehat{M}^{\prime} \rightarrow \widehat{M}$ of $\widehat{M}$, and relate our concept of guarded equation morphism to $\widehat{M}^{\prime}$ - for distinction from the "real thing" we call this notion "weakly guarded" equation morphism. At the end of our paper we will indeed verify $\widehat{M}=\widehat{M^{\prime}}+\mathrm{Id}$.

\section{Notation 4.5.}

1. We denote by $\rho: M \widehat{M} \rightarrow \widehat{M}$ the natural transformation whose components are the algebra morphisms $\rho_{Y}: M \widehat{M} Y \rightarrow \widehat{M Y}$ of the free iterative $\mathbb{M}$ algebras $\widehat{M} Y$, see Remark 2.7 .

2. The monad $\widehat{\mathbb{M}}=(\widehat{M}, \widehat{\eta}, \widehat{\mu})$ of free iterative $\mathbb{M}$-algebras has the unit $\widehat{\eta}$ given by universal morphisms of Remark 2.7 and the multiplication $\widehat{\mu}$ with $\widehat{\mu}_{Y}=\widehat{\rho_{Y}}$, see Notation 4.2 .

Remark 4.6. Recall from [7] that in every locally finitely presentable category every morphism can be factorized as a strong epimorphism followed by a monomorphism.

Definition 4.7. We define the subfunctor $\widehat{M^{\prime}}$ of $\widehat{M}$ to be the image of the natural transformation $\rho \cdot m \widehat{M}: M^{\prime} \widehat{M} \rightarrow \widehat{M}$. More precisely, for every object $X$ we have a strong epimorphism $\gamma_{X}$ and a monomorphism $\widehat{m}_{X}$ such that

$$
\rho_{X} \cdot m_{\widehat{M} X}=\widehat{m}_{X} \cdot \gamma_{X}
$$

holds. Obviously, $\gamma: M^{\prime} \widehat{M} \rightarrow \widehat{M}^{\prime}$ and $\widehat{m}: \widehat{M^{\prime}} \rightarrow \widehat{M}$ are natural transformations with $\widehat{m} \cdot \gamma=\rho \cdot m \widehat{M}$. 
Definition 4.8. A rational equation morphism $e: X \rightarrow \widehat{M}(X+Y)$ is called weakly guarded if it factorizes through $\left[\widehat{m}_{X+Y}, \widehat{\eta}_{X+Y} \cdot \mathrm{inr}\right]: \widehat{M}^{\prime}(X+Y)+Y \rightarrow$ $\widehat{M}(X+Y)$ as shown below:

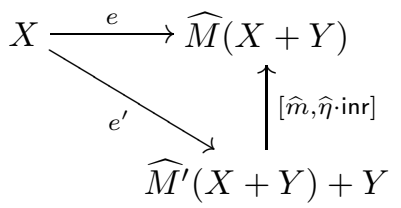

Theorem 4.9. In every iterative $\mathbb{M}$-algebra every weakly guarded rational equation morphism has a unique solution.

Sketch of Proof. This result generalizes Theorem 4.6 of [3. Suppose we are given an iterative $\mathbb{M}$-algebra $(A, a)$ and a weakly guarded rational equation morphism $e$ as in (4.3). Since $\gamma_{X+A}: M^{\prime} \widehat{M}(X+A) \rightarrow \widehat{M}^{\prime}(X+A)$ is a strong epimorphism we have, by Assumption 2.1, a morphism $s: \widehat{M}^{\prime}(X+A) \rightarrow M^{\prime} \widehat{M}(X+A)$ with $\gamma_{X+A} \cdot s=$ id. We define $e_{0}=(s+A) \cdot e^{\prime}: X \rightarrow M^{\prime} \widehat{M}(X+A)+A$. Now apply Theorem 3.9 and use the fact that $M^{\prime}$ is finitary to see that $M^{\prime} \widehat{M}(X+A)+A=$ $\operatorname{colim}\left(M^{\prime} \mathrm{Eq}_{X+A}+A\right)$. Thus, by the finite presentability of $X$, there exists an object $g: W \rightarrow M(W+X+A)$ in $\mathrm{EQ}_{X+A}$ and a factorization $w^{\prime}: X \rightarrow M^{\prime} W+A$ of $e_{0}$ through the colimit injection $M^{\prime} g^{\sharp}+A$, i. e., $e_{0}=\left(M^{\prime} g^{\sharp}+A\right) \cdot w^{\prime}$. Let $w=\left(m_{W}+A\right) \cdot w^{\prime}: X \rightarrow M W+A$ and define an equation morphism $\langle e\rangle:$ $W+X \rightarrow M(W+X+A)$ in $\mathrm{EQ}_{A}$ by its components as follows

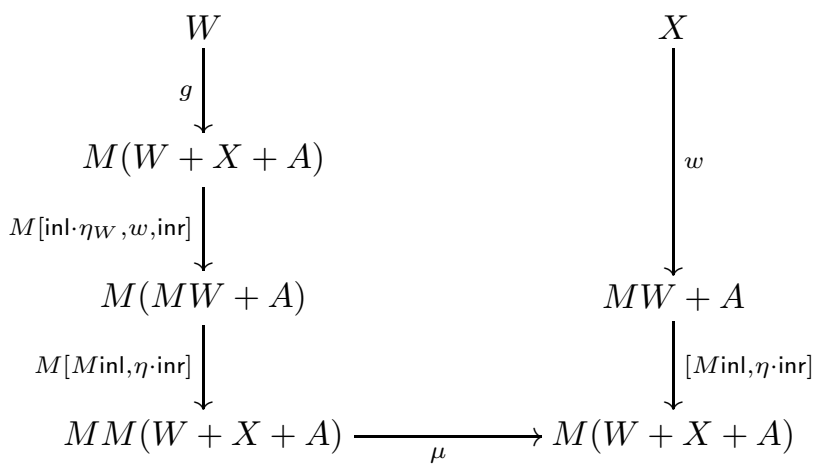

One readily proves that $\langle e\rangle$ is a guarded equation morphism, and therefore there exists a unique solution $\langle e\rangle^{\dagger}: W+X \rightarrow A$. Now let $e^{\ddagger}=\langle e\rangle^{\dagger} \cdot \operatorname{inr}: X \rightarrow A$. The technical part of the proof is the verification that $e^{\ddagger}$ is a unique solution of $e$.

\section{The Iterative Reflection}

We are ready to prove that for every ideal monad $\mathbb{M}$ the monad $\widehat{\mathbb{M}}$ of free iterative algebras (see Remark 2.7) is the free iterative reflection. More detailed: 
(1) $\widehat{M}=\widehat{M}^{\prime}+$ Id with coproduct injections $\widehat{m}$ (Definition 4.7) and $\widehat{\eta}$,

(2) the multiplication $\widehat{\mu}$ has a restriction $\widehat{\mu}^{\prime}: \widehat{M^{\prime}} \widehat{M} \rightarrow \widehat{M}^{\prime}$,

(3) every guarded equation morphism $e: X \rightarrow \widehat{M}(X+A)$ has a unique solution,

(4) the natural transformation

$$
\kappa \equiv M \stackrel{M \widehat{\eta}}{\longrightarrow} M \widehat{M} \stackrel{\rho}{\longrightarrow} \widehat{M}
$$

is an ideal monad morphism, and

(5) $\kappa$ has the following universal property: for every ideal monad morphism $\lambda: \mathbb{M} \rightarrow \mathbb{S}$, where $\mathbb{S}$ is an iterative monad, there exists a unique ideal monad morphism $\bar{\lambda}: \widehat{\mathbb{M}} \rightarrow \mathbb{S}$ with $\bar{\lambda} \cdot \kappa=\lambda$.

We have to leave (1) to the end and prove the other properties first. We will use the same terminology as in Section 4; in (3) we speak about weakly guarded equation morphisms meaning those with a factorization as in (4.3). In (4) and (5) we use the following notion of weakly ideal monads.

\section{Definition 5.1}

1. A weakly ideal monad consists of a finitary monad $\mathbb{M}=(M, \eta, \mu)$, a finitary subfunctor $m: M^{\prime} \hookrightarrow M$, and a natural transformation $\mu^{\prime}$ such that the square 2.1) commutes.

2. Suppose we have two weakly ideal monads $\mathbb{M}=\left(M, \eta, \mu, M^{\prime}, m, \mu^{\prime}\right)$ and $\overline{\mathbb{M}}=$ $\left(\bar{M}, \bar{\eta}, \bar{\mu}, \bar{M}^{\prime}, \bar{m}, \bar{\mu}^{\prime}\right)$. By a weakly ideal monad morphism we understand $a$ monad morphism $h:(M, \eta, \mu) \rightarrow(\bar{M}, \bar{\eta}, \bar{\mu})$ such that there exists a domaincodomain restriction $h^{\prime}: M^{\prime} \rightarrow \bar{M}^{\prime}$ of $h$ with $\bar{m} \cdot h^{\prime}=h \cdot m$.

3. A weakly ideal monad is called weakly iterative if every weakly guarded equation morphism has a unique solution.

Lemma 5.2. The monad $\widehat{\mathbb{M}}$ of free iterative algebras for $\mathbb{M}$ is weakly ideal.

Proof. We only need to supply the restriction $\widehat{\mu}^{\prime}: \widehat{M}^{\prime} \widehat{M} \rightarrow \widehat{M}^{\prime}$ of the monad multiplication $\widehat{\mu}: \widehat{M} \widehat{M} \rightarrow \widehat{M}$. Then $\widehat{\mathbb{M}}=\left(\widehat{M}, \widehat{\eta}, \widehat{\mu}, \widehat{M}^{\prime}, \widehat{m}, \widehat{\mu}^{\prime}\right)$ is a weakly ideal monad.

Observe first that the diagram

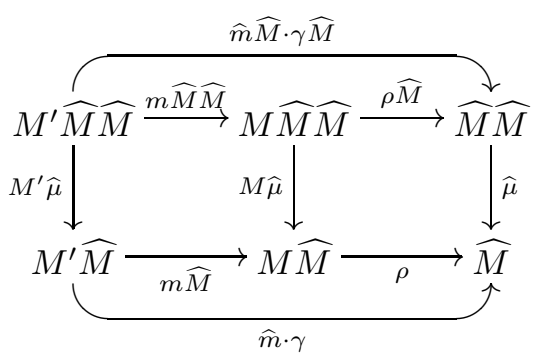

commutes. In fact, the left-hand square commutes by naturality of $m$, the rest follows from Notation 4.5 and Equation (4.2). Thus, by diagonal fill-in there exists a unique natural transformation $\widehat{\mu}^{\prime}: \widehat{M}^{\prime} \rightarrow \widehat{M}$ such that the diagram 


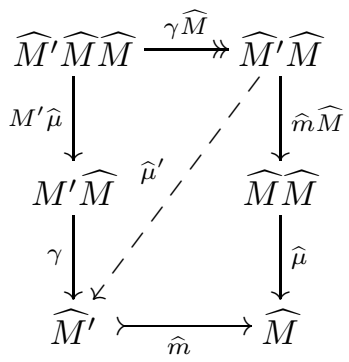

commutes. The lower triangle shows that $\mu^{\prime}$ is the required restriction of $\mu$ (cf. (2.1)).

Lemma 5.3. The monad $\widehat{\mathbb{M}}$ of free iterative algebras for $\mathbb{M}$ is weakly iterative.

Proof. It is trivial to see that Proposition 2.11 extends to weakly ideal monads. Then the desired result follows from Theorem 4.9.

\section{Theorem 5.4}

1. The natural transformation $\kappa: M \rightarrow \widehat{M}$ is a weakly ideal monad morphism.

2. Let $\mathbb{S}$ be an iterative monad. For every weakly ideal monad morphism $\lambda$ : $\mathbb{M} \rightarrow \mathbb{S}$ there exists a unique weakly ideal monad morphism $\bar{\lambda}: \widehat{\mathbb{M}} \rightarrow \mathbb{S}$ with $\lambda=\bar{\lambda} \cdot \kappa$.

Sketch of Proof. We omit the proof of item 1 and we sketch the proof of item 2.

For every object $Y$, we show that $S Y$ is an iterative $\mathbb{M}$-algebra. In fact, since $\lambda: M \rightarrow S$ is a monad morphism we obtain an $\mathbb{M}$-algebra $\mu_{Y}^{S} \cdot \lambda_{S Y}: M S Y \rightarrow S Y$. Suppose that $e: X \rightarrow M(X+S Y)$ is a guarded equation morphism. Then we form an equation morphism $\bar{e}$ with respect to the iterative monad $\mathbb{S}$ as follows:

$$
X \stackrel{e}{\longrightarrow} M(X+S Y) \stackrel{\lambda *\left(\eta_{X}^{S}+S Y\right)}{\longrightarrow} S(S X+S Y) \stackrel{\mu^{S} \cdot S \mathrm{can}}{\longrightarrow} S(X+Y) .
$$

It is not difficult to see that $\bar{e}$ is guarded, and that there is a 1-1 correspondence between solutions of $e$ in the algebra $S Y$ and solutions of $\bar{e}$ with respect to $\mathbb{S}$. Since the latter exists uniquely, so does the former. Now the freeness of $\widehat{M} Y$ as an iterative algebra implies the existence of a unique homomorphism $\bar{\lambda}_{Y}$ of $\mathbb{M}$-algebras from $\left(\widehat{M} Y, \rho_{Y}\right)$ to $\left(S Y, \mu_{Y}^{S} \cdot \lambda_{S Y}\right)$ such that $\bar{\lambda}_{Y} \cdot \widehat{\eta}_{Y}=\eta_{Y}^{S}$. One then proves that $\bar{\lambda}$ is a weakly ideal monad morphism with $\lambda=\bar{\lambda} \cdot \kappa$, and that $\bar{\lambda}$ is uniquely determined.

Theorem 5.5. The iterative reflection of an ideal monad $\mathbb{M}$ is the monad $\widehat{\mathbb{M}}$ of free iterative $\mathbb{M}$-algebras.

Proof. In view of the preceding results this amounts to proving that $\widehat{M}=\widehat{M}^{\prime}+\mathrm{Id}$ with injections $\widehat{m}$ and $\widehat{\eta}$.

It is known that every weakly ideal monad $\mathbb{S}$ has an ideal coreflection $c: \mathbb{S}^{*} \rightarrow$ $\mathbb{S}$ and $\mathbb{S}^{*}$ is iterative whenever $\mathbb{S}$ is weakly iterative (see [3], Proposition 6.7). More 
detailed: let $\mathbb{S}$ be weakly ideal with the corresponding subfunctor $s: S^{\prime} \hookrightarrow S$. Then for the functor $S^{*}=S^{\prime}+$ Id there is a structure of a monad $\mathbb{S}^{*}$ with unit inr : Id $\rightarrow S^{\prime}+$ Id and multiplication $\mu^{*}: S^{*} S^{*} \rightarrow S^{*}$ such that the morphism $c=[s, \eta]: S^{\prime}+\mathrm{Id} \rightarrow S$ is a weakly ideal monad morphism from $\mathbb{S}^{*}$ to $\mathbb{S}$. Moreover, every weakly ideal monad morphism from an ideal monad into $\mathbb{S}$ uniquely factorizes through $c$. We now apply this to $\mathbb{S}=\widehat{\mathbb{M}}$ : we obtain an iterative monad $\widehat{\mathbb{M}}^{*}=\left(\widehat{M}^{\prime}+\mathrm{Id}\right.$, inr, $\left.\widehat{\mu}^{*}\right)$ and a weakly ideal monad morphism $c=[\widehat{m}, \widehat{\eta}]: \widehat{\mathbb{M}}^{*} \rightarrow \widehat{\mathbb{M}}$. We prove that $c$ is an isomorphism-this implies the desired statement $\widehat{M}=\widehat{M^{\prime}}+\mathrm{Id}$.

Since $\mathbb{M}$ is an ideal monad, the weakly ideal monad morphism $\kappa: \mathbb{M} \rightarrow \widehat{\mathbb{M}}$ factorizes as $\kappa=c \cdot \kappa^{*}$ for an ideal monad morphism $\kappa^{*}: \mathbb{M} \rightarrow \widehat{\mathbb{M}}^{*}$. By the universal property of Theorem 5.4 we obtain an ideal monad morphism $d: \widehat{\mathbb{M}} \rightarrow$ $\widehat{\mathbb{M}}^{*}$ such that $d \cdot \kappa=\kappa^{*}$. Then we get $c \cdot d \cdot \kappa=\kappa$ from which we immediately conclude that $c \cdot d=\mathrm{id}$. Now $d \cdot c$ is an ideal monad endomorphism on the ideal coreflection $\widehat{M^{\prime}}+\operatorname{Id}$ of $\widehat{M}$. Thus, the equality $c \cdot d \cdot c=c$ proves that $d \cdot c=\mathrm{id}$. This establishes that $\widehat{M}$ is a coproduct of $\widehat{M}^{\prime}$ and Id with injections $\widehat{m}: \widehat{M}^{\prime} \rightarrow \widehat{M}$ and $\widehat{\eta}: \mathrm{Id} \rightarrow \widehat{M}$ as desired.

Corollary 5.6. The full embedding of the category $\operatorname{IFM}(\mathcal{A})$ of iterative monads to the category $\mathrm{FM}_{\mathrm{id}}(\mathcal{A})$ of ideal monads has a left adjoint.

\section{Conclusions and Future Research}

For every ideal monad $\mathbb{M}$ of Set we proved that the monad $\widehat{\mathbb{M}}$ of free iterative Eilenberg-Moore algebras for $\mathbb{M}$ is iterative. In fact, $\widehat{\mathbb{M}}$ is the iterative reflection of $\mathbb{M}$. We thus derive a number of examples of iterative monads, cf. Examples 2.8.

1. For the finite list monad $M X=X^{*}$ we obtain the iterative reflection $\widehat{M} X=$ $X^{*} \cup\{\perp\}$ where $\perp$ is an absorbing element.

2. Analogously, for the finite bag monad $\mathbb{M}$ we have $\widehat{M} X=M X \cup\{\perp\}$ where $\perp$ is an absorbing element.

3. For the finite tree monad $\mathbb{M}$, the reflection is the monad $\widehat{\mathbb{M}}$ of rational trees.

4. An analogous example works for non-ordered finite trees: here $\widehat{\mathbb{M}}$ is the monad of rational unordered trees. This follows from results in [2].

5. The iterative reflection of the unary algebra monad $M X=X \times \Sigma^{*}$ is the $\operatorname{monad} \widehat{M} X=X \times \Sigma^{*}+\Sigma^{*}\left(\Sigma^{*}\right)^{\omega}$.

Although the existence of iterative reflections was established in [5] for all ideal monads on extensive, locally finitely presentable categories, in the present paper we restricted our attention to monads in Set-like categories. The reason was purely technical: at two stages, in the proofs of Lemma 3.8 and Theorem 4.9, we needed the hypothesis that every strong epimorphism splits. We do not know whether our results hold in general extensive, locally finitely presentable categories. And for the development of our paper those results seem indispensable. The generalization of our results to a larger collection of base categories is therefore left as an open problem. 
In the future we intend to work on a connection of our results to iteration as applied in process algebra.

Acknowledgment. We are grateful to one referee who suggested several improvements of the presentation of our paper.

\section{References}

1. Adámek, J., Börger, R., Milius, S., Velebil, J.: Iterative algebras: How iterative are they? Theory Appl. Categ. 19, 61-92 (2008)

2. Adámek, J., Milius, S.: Terminal coalgebras and free iterative Theories. Inform. and Comput. 204, 1139-1172 (2006)

3. Adámek, J., Milius, S., Velebil, J.: Iterative algebras at work. Math. Structures Comput. Sci. 16.6, 1085-1131 (2006)

4. Adámek, J., Milius, S., Velebil, J.: Elgot algebras. Logical Methods Comput. Sci. 2(5:4), 31 (2006)

5. Adámek, J., Milius, S., Velebil, J.: Iterative reflections of monads. Math. Structures Comput. Sci. (accepted for publication)

6. Adámek, J., Milius, S., Velebil, J.: A description of iterative reflections of monads, http://www.stefan-milius.eu

7. Adámek, J., Rosický, J.: Locally presentable and accessible categories. Cambridge University Press, Cambridge (1994)

8. Barr, M.: Coequalizers and free triples. Math. Z. 116, 307-322 (1970)

9. Elgot, C.C.: Monadic computation and iterative algebraic theories. In: Rose, H.E., Shepherdson, J.C. (eds.) Logic Colloquium 1973. North-Holland Publishers, Amsterdam (1975)

10. Elgot, C.C., Bloom, S., Tindell, R.: On the algebraic structure of rooted trees. J. Comput. System Sci. 16, 361-399 (1978)

11. Gabriel, P., Ulmer, F.: Lokal präsentierbare Kategorien. Lecture N. Math., vol. 221. Springer, Berlin (1971)

12. Ginali, S.: Regular trees and the free iterative theory. J. Comput. System Sci. 18, 228-242 (1979)

13. Mac Lane, S.: Categories for the working mathematician, 2nd edn. Springer, Heidelberg (1998)

14. Nelson, E.: Iterative algebras. Theoret. Comput. Sci. 25, 67-94 (1983)

15. Tiuryn, J.: Unique fixed points vs. least fixed points. Theoret. Comput. Sci. 12, 229-254 (1980) 